\title{
From the finger lift to the palm-up open hand when presenting a point: A methodological exploration of forms and functions
}

\author{
Alan Cienki ${ }^{1,2}$ \\ 1 Vrije Universiteit Amsterdam, Amsterdam, the Netherlands \\ 2 Moscow State Linguistic University, Moscow, Russia \\ Corresponding author: Alan Cienki (a.cienki@vu.nl)
}

Academic editor: Cornelia Müller • Received 19 May 2021 • Accepted 22 July 2021 • Published 25 October 2021

\begin{abstract}
There are many studies on the palm-up open hand (PUOH) as a gesture used when the speaker is presenting a point, but many other gesture forms can also accompany this discursive move. While the forms may appear diverse based on traditional means of gesture analysis, the relations between a number of them can be analyzed in a coherent way using the kinesiological system developed by Boutet (2010; 2018; to appear). This system approaches gestural forms not from external criteria based on the viewer's perspective (involving hand shapes, locations in gesture space, etc.), but rather from the inside; in the present analysis, the focus is on the directions of movements made at joints in producing them (e.g. whether flexion vs. extension was involved, whether any rotation involved was inward or outward, etc.). Four particular gestures are considered as points along a continuum: from the finger extension, to the forearm and wrist turn-out of the hand, to the supination of the PUOH, to an exaggerated form of the PUOH produced with extension and abduction of the upper arm. A multifunctional model is also proposed to analyze the degree of transparency of the different gestures' representational, pragmatic, and interactive functions. The functional analysis performed with this model is grounded in form features from a combination of the kinesiological and traditional four-parameter form-based systems. This methodological exploration provides a model which could be applied or adapted for the analysis of other groups of gestures that are related in terms of their physiological means of production.
\end{abstract}

\section{Key Words}

gesture forms, gesture functions, kinesiological system, methodology, palm-up open hand, pragmatic gestures, Russian

\section{Introduction}

A number of studies have pointed out the role that gesture can play when speakers are presenting a point, introducing a topic in their discourse, or offering an idea for consideration (e.g., Bavelas, 1994; Calbris, 2011; Cooperrider et al., 2018; Streeck, 2009; and other works cited throughout this article). For example, in his analysis of material from Italian and English, Kendon (2004, p. 266) characterizes the "Palm Presentation" gesture as follows: it "is typically used in association with passages in the verbal discourse which serve as an introduction to something the speaker is about to say, or serve as an explana- tion, comment or clarification of something the speaker has just said." Müller (2004) devotes an entire chapter to the gesture that "is characterized by a specific hand shape and orientation: palm open, fingers extended more or less loosely, palm turned upwards" (p. 233).

However, there is actually a range of gestural forms that are used when speakers are presenting a point-from turning the hand outward to varying degrees such that the palm is not even turned up and the fingers of the hand are only half-open, to simply lifting a finger momentarily. What I would like to argue is that among speakers of a number of European languages (and here we will focus mainly on Russian), these 'presenting' gestures can be 
characterized according to a coherent framework, despite the range of variation in their forms and functions. I will argue (a) that the forms can be seen as forming a continuum in terms of the different degrees of exertion involved in their production. (b) The range shown by their forms can be analyzed in a coherent way through the kinesiological system characterized by Boutet (2010 and elsewhere). And (c) the uses of these differing forms can be characterized in terms of several functions they exhibit simultaneously, but to different degrees of transparency with which the use of that function can be interpreted. Proceeding through this analysis will serve as a methodological demonstration of the value of combining two types of gesture form analysis - one kinesiological, and the other based on a set of four descriptive form parameters - along with a novel, graded form of gesture function analysis.

Since it appears that the claims to be made here apply to other cultures beyond Russian, the present study can constitute a starting point which can be tested against gesture use with other languages in the future. In addition, the method of analysis that is presented here offers a combination which could be useful to test on a wider scale in the future to assess the reliability with which it can be used. We will now turn to the specifics of the forms and functions of these gestures.

\section{Kinds of gestures considered in past studies as presenting a point}

In past research, the palm-up open hand (PUOH) has been discussed as the prime example of a manual gesture that accompanies a speaker's presentation of a point. Müller (2004) provides not only an analysis of different uses of this gestural form as used by speakers of German, but also a concise history of how the gesture has been discussed in the past (and see also Kendon, 2004 on this). Müller (2004), in fact, identifies a number of functions beyond presenting a point that have been discussed for the PUOH, but for most of these an additional formal element is entailed, such as listing a series of arguments with repeated downward motion of the $\mathrm{PUOH}$, or indicating a wide range of ideas being offered with wide lateral motion of the PUOH (p. 252). (See Cooperrider et al., 2018, for a cross-cultural review of the gesture's various functions.) Here we will restrict our starting point for inquiry to the 'simple' palm-opened hand, held momentarily in a stationary position, usually in front of the speaker, used with the function of presenting a point.

Even limiting ourselves to this form and function, other studies have considered this type of $\mathrm{PUOH}$ as part of a broader category of "pragmatic gestures" (as in Kendon, 2004). Streeck (2009, ch. 8) considers the PUOH in the context of various discourse-related gestures which accomplish what he calls "speech handling", e.g. offering a central idea or new information to the interlocutor, and in turn, waiting to receive a response from them - an analysis which Müller (2004, p. 234) notes goes back to Quintilian in his description of Roman oratorical gestures. Calbris (2011, pp. 331-332) points out (on the basis of French data) that it is not just about the open hand, but the opening of the hand. That is, the opening of the hand goes with the offering of an explanation or the presenting of a point (it is part of a process). Thus, many researchers highlight the interactive nature of the PUOH (and Bavelas, 1994, explicitly categorizes it as an "interactive gesture"), but we see that it also involves elements that have to do with schematic presentation of the topic being spoken of. This metaphoric element of the gesture's use will be discussed further below. It has been observed to be a recurrent gesture across a number of European languages and cultures, according to the definition of Bressem and Müller (2014, p. 1576): a recurrent gesture is one that "is used repeatedly in different contexts and its formational and semantic core remains stable across different contexts and speakers" (see also Müller, 2010, p. 41). This stability, though, does not mean an absolute fixedness of either the formal or functional side of the gesture as a symbol; as Bressem and Müller point out, some variation is allowable in how recurrent gestures are produced, and the range of meanings encompassed by the semantic core has some flexibility, whereby the specific meaning is dependent on the speech context (pushing the "semantic" core more to pragmatic uses). In that regard, recurrent gestures (including the PUOH) are different from emblems (Efron, 1972/1941), in which a conventionally quite fixed gesture form corresponds more clearly to a particular meaning/ function within a given culture.

However, other gestures that can serve the function of presenting a point have not even been recognized in the literature. One example of these is what may have been considered as self-adapters. Adapters are commonly known as (usual manual) actions that "fix oneself" in some way. These self-directed, "body-focused movements" (Freedman, 1972, p. 155) can involve adjusting something on oneself (like one's glasses or one's clothing) or adjusting one's cognitive and/or emotional state, helping focus oneself mentally or sooth oneself emotionally, e.g. by rubbing oneself with one's fingers or rubbing one's fingers together. In the latter case, such self-adapters can also be seen as indicating stress or nervousness.

Yet there can be more transpiring within self-adapter movements than just the functions mentioned above. Some involve additional elements which diverge from self-directed movement and momentarily go outward, and do so in relation to the content and rhythm of the speech. A current study on the gestures made by simultaneous interpreters at work ("Verbal and co-verbal behavior under cognitive pressure", supported by the Russian Science Foundation) has revealed many instances of self-adapters - which in itself may not be surprising, given the cognitive stress level that can be experienced when conducting this work. But close analysis of these self-adapters, facilitated by video recordings made with a small GoPro camera on the desk facing the interpreter, 
revealed small movements that also accompany the presentation of points in the discourse. A main category here involves finger movements extending outward and then returning back to their original position. A prime example was when speakers' hands were folded together on the desk in front of them, and they raised one or both thumbs when making a point, often holding it/them up in raised position while stating the point, and then lowering it or them back down upon completion of the statement (Figure 4, below, provides an example of such a gesture).

While the PUOH and the self-adapters with additional movements might be seen as involving quite different behaviors, the relevance of the differences between them may largely be a factor of how they are being analyzed, as the following section will make clear.

\section{Analysis of the forms, part 1: Description using the traditional four parameters}

What is proposed here is that the forms in question can be seen as part of a continuum. Here we will illustrate four points on the continuum in Figures 1-4 as reference points for characterizing it, and will describe them in more detail below. As will be evident, we will generally be concerned with gestures produced with one hand, as two-handed gestures with palms open can serve various other functions (as discussed in Kendon, 2004; Müller, 2004; and elsewhere).

The way of describing manual gestures that has become established in gesture studies derives from a system developed for analyzing manual forms used in sign languages (Stokoe, 1960) and which became widespread in gesture studies with its use in McNeill (1992). Bressem (2013) provides a detailed elaboration of the system. It consists of four parameters: (1) handshape; (2) orientation of the palm of the hand (the direction it is facing), sometimes supplemented with a characterization of the direction in which the fingers are oriented (especially if the fingers are not in a flat plane with the palm of the hand); (3) direction and manner of motion of the hand(s); and (4) location in which the gesture was produced in relation to the front of the speaker. For this, many adopt the grid used in McNeill's research, distinguishing, for example, the central space as a kind of square in front of the speaker's torso, a more peripheral space at the sides of the body and by the shoulders and waist, and an extreme periphery in the space further out to the sides, above the head, and below the waist.

On the one end of the continuum we can begin with an extreme example of the palm-up open hand as a presentation gesture, shown in Fig. 1. In this case, we are dealing with the moment when a theatrical magician has completed a trick, usually which results in something surprisingly appearing or disappearing. The magician completes the performative act of presentation of this result

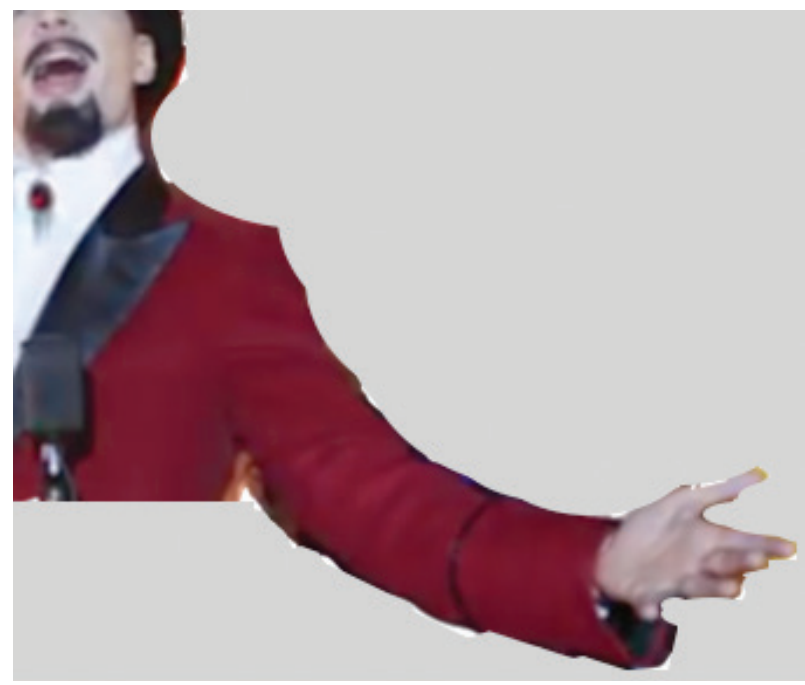

“Tele [Dva]!” ' Tele Two'

Figure 1. "Ta-daa"-type extreme example of palm-up open hand presentation (Square brackets will be used in the transcriptions of speech to mark the syllable coinciding with the stroke phase of the gesture shown).

with a palm-up open hand gesture, produced with an outstretched arm extended some distance from the speaker's torso, either in front of, or off to the same side of, the speaker as the hand that is doing the presenting. Usually this is accompanied by an exclamation, "Ta-daa!" The example shown was part of a marketing campaign for the telecommunications company TELE2 (which can be seen here: https:/www.youtube.com/watch? $\mathrm{v}=\mathrm{R} 5 \mathrm{bKVhCMb}$ tM). In this case, it was in Croatia, but the 'performance' of such a gesture in the context of a magician's act is one familiar across many American and European cultures, and thus is being included here. The presenter makes the gesture when announcing the name of the company (in Croatian), "Tele Dva!" 'Tele two'. He then repeats the gesture, directed more towards the camera, as the other performers behind him exclaim "Ta-daa!" The extreme nature of the presentation carries over to the function of the gesture (discussed further below), in that it is not a mere presentation of a speaker's idea, but rather an actual transformation that the audience just witnessed-that is, not a presentation of a physical thing necessarily (not always a rabbit produced out of a hat), but of the point that the trick was successful, signaling that the result should be appreciated by the audience. In this sense, the function relates to the more classic palm-up open hand gesture used in conversation to present a point to the listening and viewing interlocutor for their consideration.

Next on this continuum of gestures presenting a point is the "classic" palm-up open hand, as found in the examples noted in the previous section, and as illustrated in Fig. 2. Fig. 2A shows the more fully produced form, with the palm fully facing up, while Fig. 2B shows a frequent instantiation of it, with the palm not completely turned upward. In Fig. 2A (adapted from Iriskhanova \& Cienki, 
2018, p. 31) the Russian speaker in a conversation with a friend picks up on her interlocutor's point and adds her own; in Fig. 2B the Russian television news anchor comments on a story by asking a rhetorical question.

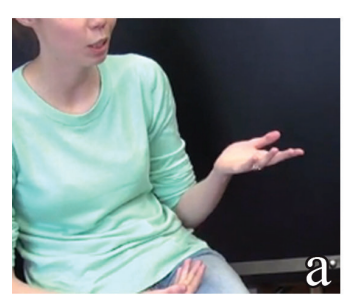

"На [сам]ом деле" 'In fact'

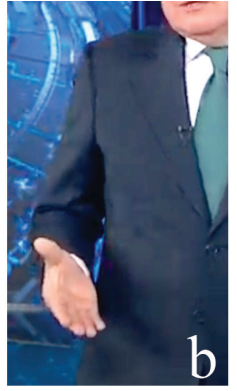

“то, [что́] тогда вмешательство?” 'then what is interference?'
Figure 2. More common examples of palm-up open hand.

Kendon (2004, p. 265) describes the gesture as involving "a wrist extension, often combined with a slight lowering of the hand, and followed by a hold." Most prototypically, it is produced in front of the speaker, closer to the body than the "magician's presentation", described above. Producing the PUOH further out from the body (and not even as far out as in Figure 1) involves a different use of this form-more likely for presenting a physical object in the speaker's presence. Furthermore, while the fingers of the hand are known to often point toward the interlocutor, we are excluding from discussion here $\mathrm{PUOH}$ gestures which involve movement along the axis of the fingers, pointing towards someone or something. (On this, see Kendon's [2004, ch. 11] discussion of PUOH pointing gestures, and Grishina [2017, ch. 2] specifically on PUOH pointing gestures in Russian.)

The next two categories concern the smaller and less effortful instantiations of presentation gestures. A prime example involves what can be called a turn-out of the hand, as shown in Figure 3. As in the present case, where a Russian politician is making a statement during a televised meeting, this might be found when the speaker is seated with their hands on a table in front of them, palms facing down or facing each other/the center space. The hand turns out from the lower arm, but stops before reaching a palm-up position, and then returns back to its original position. It is what Ferré (2011) refers to as a "hand flip". In some ways this type fits Freedman's (1972) characterization of what he called a "minor qualifier": "these movements tend to be small, involving often simply a turning of the wrists, well delineated, but not staccato-like movements" (p. 159). A related instantiation can be produced when the speaker is standing, with the arms hanging down, where the turn-out is initiated by the thumb turning outwards with the rest of the hand following to some degree. This might include a simultaneous slight lifting of the hand and lower arm. The retraction involves a turning back of the hand toward the self and a return to the rest position.

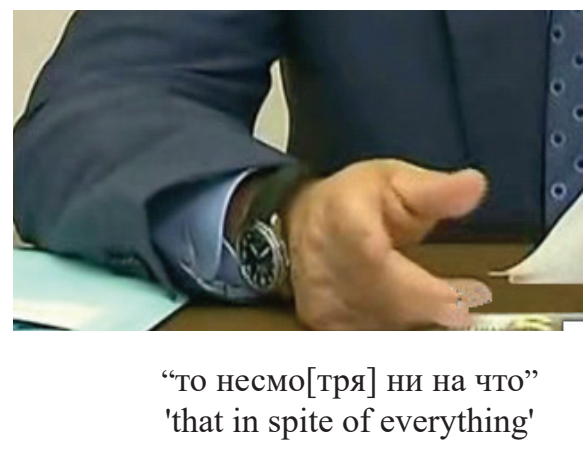

Figure 3. The turn-out.

The last point on the continuum concerns various forms of finger lifts, most often produced when the speaker is seated, with their hands in front of them on a table, palms down or facing the speaker. It can involve the extension of a single finger (such as the index finger) followed by its retraction. If the speaker's hands are folded, it can consist of lifting one or both thumbs. Fig. 4 shows a lift of one thumb and a partial lift of the other from this folded-hands position. The speaker is a simultaneous interpreter, a native speaker of Russian who is listening to a lecture in English and interpreting it into Russian. He is alone in an interpreting booth and there is no one visible in the room in front of him that he is looking into. The finger-lift gesture shown accompanies the stressed word that is the Russian equivalent for "let's" in the phrase "let's forget about that", pragmatically accompanying the prosodic stress on this word of the phrase that he is interpreting. In this regard, this kind of small biphasic (out-in) movement at this end of the continuum fades into one type of 'beat' gestures, described by McNeill (1992) and others, which provide emphasis to the point the speaker is making.

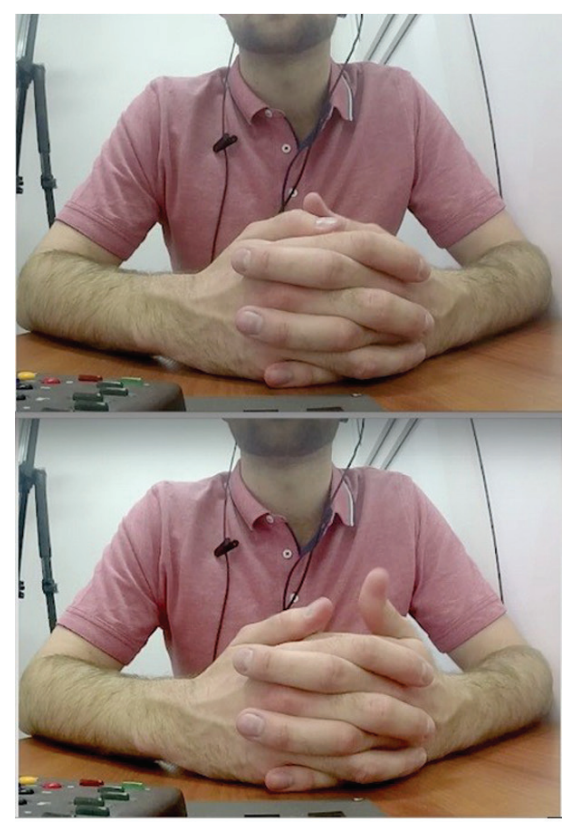

"но [давайте за]будем .. об этом" 'but let's forget .. about that'

Figure 4. Finger lift. 


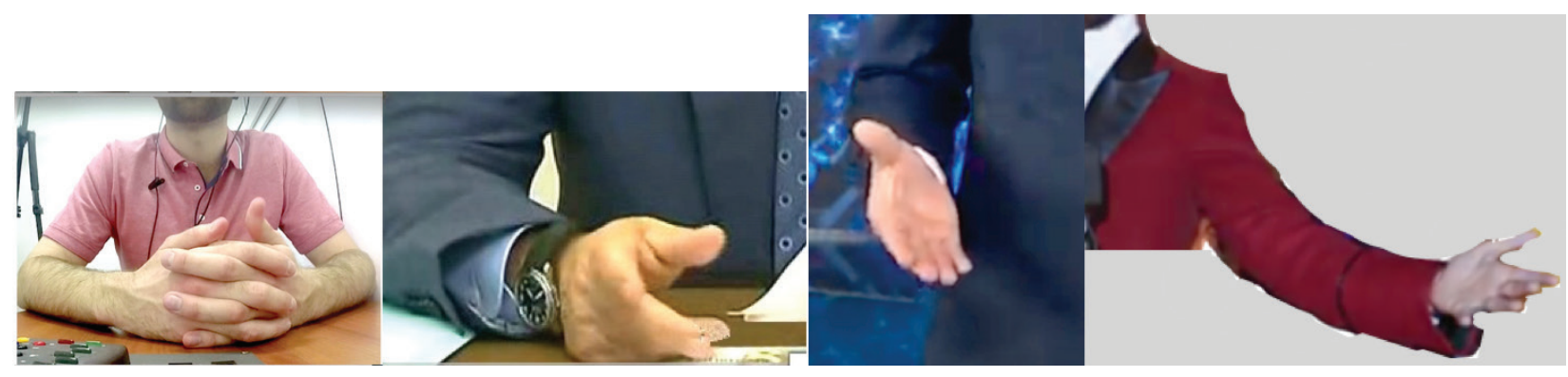

Figure 5. Points on the continuum of gestures presenting a point.

Such gestures have not been taken into consideration in many previous studies in so far as research on manual gestures primarily focusses on free-hand gestures; any movements made while the hands are folded and touching each other might normally be categorized as forms of self-adapters and, therefore, not be taken into consideration in such studies. However, it should be noted that the finger-lift movements here are not ones that fit the usual definitions of self-adapters: they are outward movements, not self-oriented self-touching movements, even if they are possibly embedded in a posture of one hand touching the other.

The continuum as a whole can be represented by these gestures as reference points along it, as in Figure 5.

What we can note is that if the gestures described above were characterized in terms of all four form parameters that are often used in gesture description, that would highlight the differences between the gesture forms described (considering the different orientations, locations, and forms of movement of the hands), rather than any similarity or continuity of form between them. This is because, as Boutet et al (2016) observed, the nature of the four-parameter system is one that inherently employs an external viewpoint on gestural phenomena. It involves describing the gestures' handshapes, palm orientations, locations, and movements using external frames of reference. These include shapes that we compare the gestures to (involving which fingers are straight or bent as in Bressem, 2013, or employing the handshapes of sign languages, as in McNeill, 1992); a grid for viewing the gesture space (frequently adopted from McNeill, 1992) superimposed onto the video images; palm orientation according to points of orientation outside the body (center-space, away from body, up, down, with "towards body" being the exception); and motion categories based again on shape descriptions (straight line, curved, etc.), directions of the gesture movements as viewed (e.g. diagonally up to the right), and qualities of movement (e.g. fast or slow, large or small). In sum, using the four form parameters creates a barrier to seeing possible connections between the gesture forms described above; it appears to simply present a conundrum in this case as to what, in terms of form, connects these gestures that are all used when presenting a point. However, using the kinesiological system provides us with a way to look at gesture from the inside (as noted in Boutet et al., 2016, p. 137), from how it is produced, and this can help us realize the internal logic of gesture production (what we might call a physio-logical perspective).

\section{Intermezzo in the analysis of the forms: Introduction of the kinesiological system}

This logic of gesture production from the kinesiological point of view comes from the kinesthetic perspective being taken on the gestures. First, from this perspective, it is clear that the four points on the continuum represent different degrees of exertion to produce the gestures. Here we can draw upon Laban's influential notion of effort, which has also been instrumental in Kendon's (1980; 2004) and McNeill's (1992) analyses of gesture. As summarized in Cienki (2013, p. 669), Laban and Lawrence (1974/1947, pp. 11-12) "characterize effort in terms of four factors of exertion: weight (with gesture this usually concerns exertion to overcome the relevant part of one's own body weight), space (exertion according to the path of motion followed, e.g., flexible or direct), time (speed of motion), and flow (control of the movement, as fluid versus bound)."

This attention to the parts and their movement that produce the gestures takes us to the kinesiological system for gesture description, developed by Boutet $(2010 ; 2018$; to appear) and Boutet et al. $(2016 ; 2018)$, drawing upon Kapandji (1997). The kinesiological approach involves using biological (i.e. based on the forms of human beings) and physical—biomechanical—categories for analyzing how gestures are produced. This begins by identifying the body parts that produce the gestures, called 'segments' in this framework. As defined in the summary of the kinesiological system in Boutet et al. (2018, p. 144), a segment is "a non-deformable anatomical unit situated between two articulations" (that is, between two joints). Examples are the forearm and the phalanges of the fingers; body parts that we commonly refer to often involve several segments taken together, such as the arm, the fingers, and the hand. The segments can be moved in different ways; each pattern of movement constitutes what is called a degree of freedom. "A degree of freedom is defined as an axis around which movements relative to a segment are possible" (ibid.). The degrees of freedom to be considered here with the movements they make possible are illustrated in Figure 6-9 and can be described as follows.

- Extension versus flexion: Technically, this can be described as movement pivoting at the axis that transverses the joint. In simple terms, flexion involves a bending 'inward' at a joint, as opposed to extension, 
which involves stretching that limb out. We can see flexion in the bending of the elbow, making a more acute angle between the upper and lower parts of the arm, or in the movement of the palm of the hand toward the underside of one's arm. Extension entails stretching the arm or forearm straight out; in the case of the wrist, a small amount of movement 'backward' is also possible, as shown in Fig. 6A.

A.

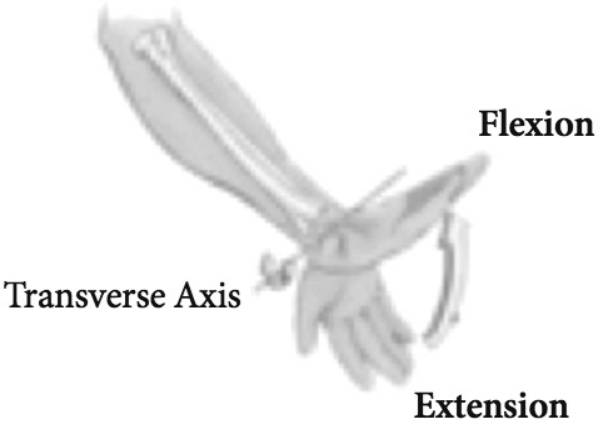

B.

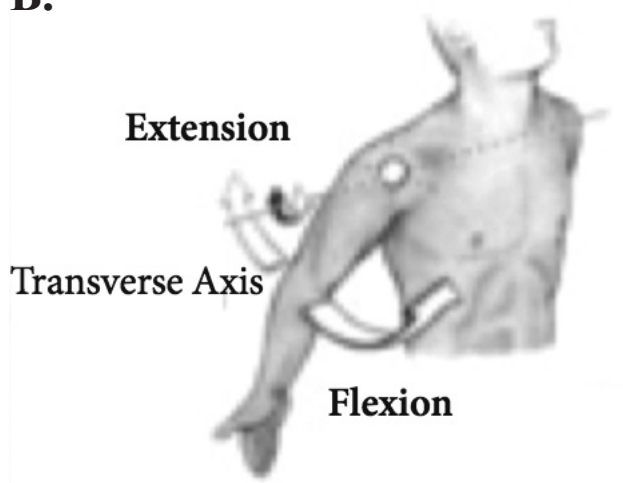

Figure 6. Extension and flexion $(\mathbf{A})$ at the wrist and $(\mathbf{B})$ at the elbow (Boutet et al., 2018, p. 145).

- Abduction versus adduction concerns the difference between movement away from (ab-) or towards (ad-) the center of the body. In the case of the elbow, adduction entails, for example, moving one's forearm across the front of one's torso, as shown in Fig. 7, and in the case of the shoulder, the abduction pivots the arm at the sagittal axis (here: the frontback axis of the body), moving the arm outward to the side, away from the torso. For another example, wagging one's finger back and forth at someone involves alternating adductive and abductive movements of the extended finger.

- Rotation is movement along the axis of a body part (a longitudinal axis), such as the upper arm from the shoulder, or the forearm from the elbow. This is a type of movement which some joints in the body allow for more than others do: contrast the wide rotation possible from the shoulder versus the more limited possibility of rotating the joints of our fingers, other than the thumb. Rotation can be interior

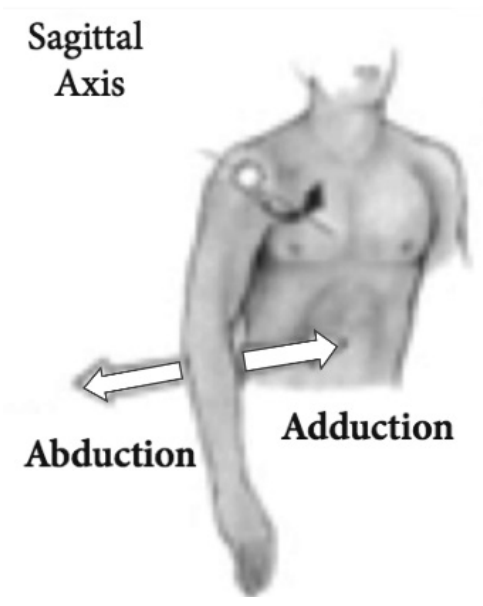

Figure 7. Abduction and adduction, here: from bending at the elbow (Boutet et al., 2018, p. 145).

or exterior in direction (inward towards or outward away from the center of the body, respectively).

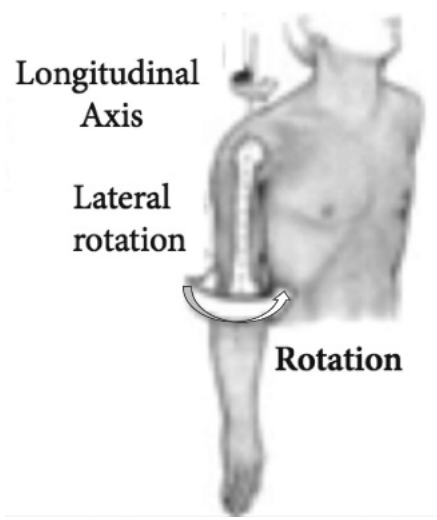

Figure 8. Rotation-interior rotation of the upper arm (Boutet et al., 2018, p. 145).

- Supination versus pronation: Supination of the hand involves exterior rotation of the hand and forearm so that the palm surface is facing upward, resulting in the hand laying on a horizontal surface on its back (supine), while pronation entails interior rotation of the hand and forearm so that the palm surface can face downward, allowing the hand to lay face down (prone) on a horizontal surface, as shown in Fig. 9.

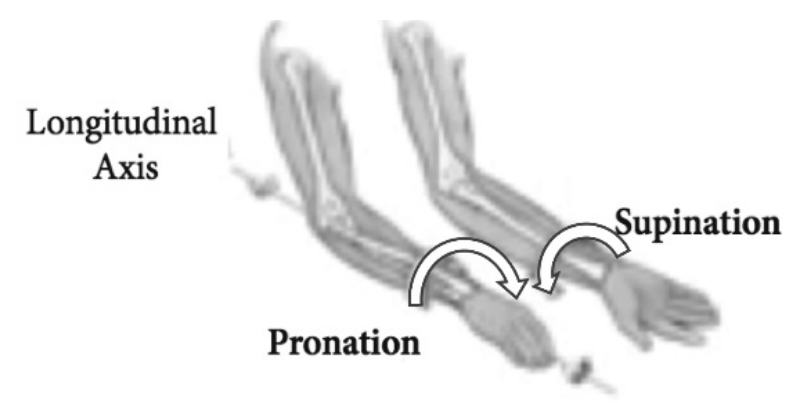

Figure 9. Pronation versus supination of the hand (Boutet et al., 2018, p. 145). 
If we consider these movement components and the order in which they are employed for the production of any gesture, we find that they provide a coherent framework for describing the different presentation gestures along a continuum of degrees of exertion required in order to produce them. A key feature of the scale of the continuum is not only that more effort is exerted as we go from the finger lifts all the way to the "ta daa" presentation gesture, but also that the effort is exerted over more articulators (bodily segments), which, consequently, involves the lifting of more weight (of one's own limbs) and the use of more space.

\section{Analysis of the forms, part 2: Description using the kinesiological system}

Starting with the smallest movements, the finger lifts involve extension of some of the smallest segments of the human body - those in the fingers. The essential, minimally necessary extension is at the joint where the finger meets the palm of the hand, with further exertion leading to extension of the other joints, maximally resulting in a fully stretched finger. Note that the extension of any finger could be at issue here - not just thumbs (as in Fig. 10): it could be the index finger alone, or another finger. It is an empirical question as to which fingers are more likely to be extended individually, but our initial evidence shows that thumb and index finger extension are certainly common. It also appears the hand can be in various palm orientations and the finger extension can still be carried out with presentation of a point in speech. Note that in the kinesiological system, there is a unified explanation for the forms, regardless of the orientation of the palm or location of the hand in space, whereas in the traditional four-parameter system of gesture description, each different finger and each different location of the hand would call for quite a different description of the gesture.

The next gestural form in the continuum from smallest to largest involves some degree of finger extension plus partial rotation of the forearm from the elbow and the wrist. The rotation in this case is outward from the sagittal

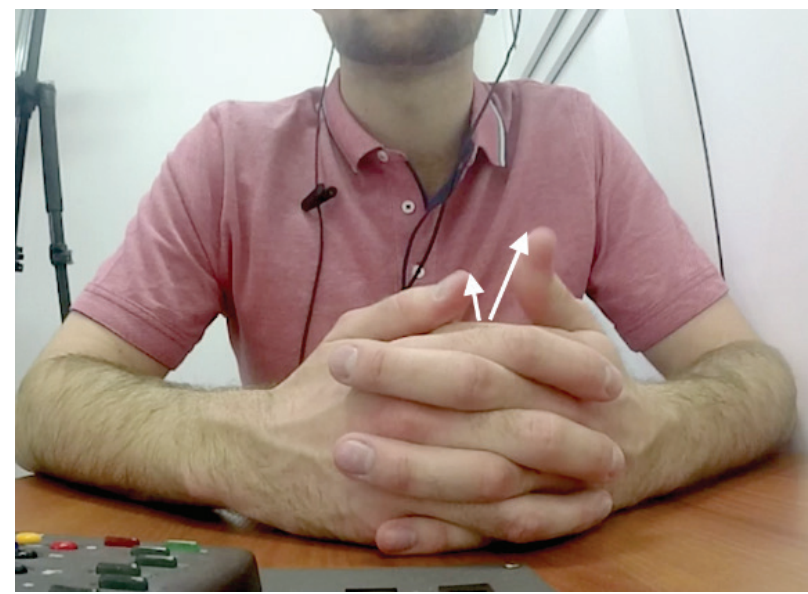

Figure 10. Extension of the two thumbs, on the left hand more than on the right hand. (front-back) plane of the body, thus abduction. This produces what we can call the hand turn-out (Fig. 11). Extension of multiple fingers produces an opening of the hand with the turn-out. Once again, the hand can be in different locations when the hand turn-out is produced, e.g. the hand could be resting on a table, or hanging down by one's side while one is standing; the same basic outward rotation for a hand turn-out can be accomplished in either position.

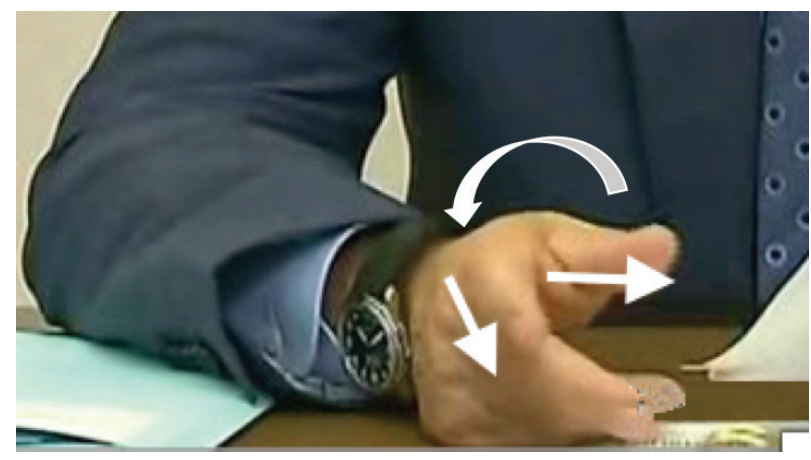

Figure 11. Hand turn-out, with extension of the thumb and forefinger (and slightly of the middle finger) and partial rotation outward of the forearm and wrist (abduction).

Continuing the rotation of the lower arm further along, with more effortful extension of the fingers, produces the opening of the hand. Maximal exertion in the lower arm rotation and finger extension results in supination of the hand, yielding the prototype of the palm-up open hand, seen above in Fig. 2A. Fig. 12 (an adaptation of Fig. 2B) shows the PUOH with partial supination.

Finally, the maximal version of presentation involves all of the above types of movement plus extension at the elbow and shoulder (producing a PUOH further out in front of oneself), along with possible abduction from the elbow and shoulder (resulting in a PUOH produced out to one's side, as in Figure 13).

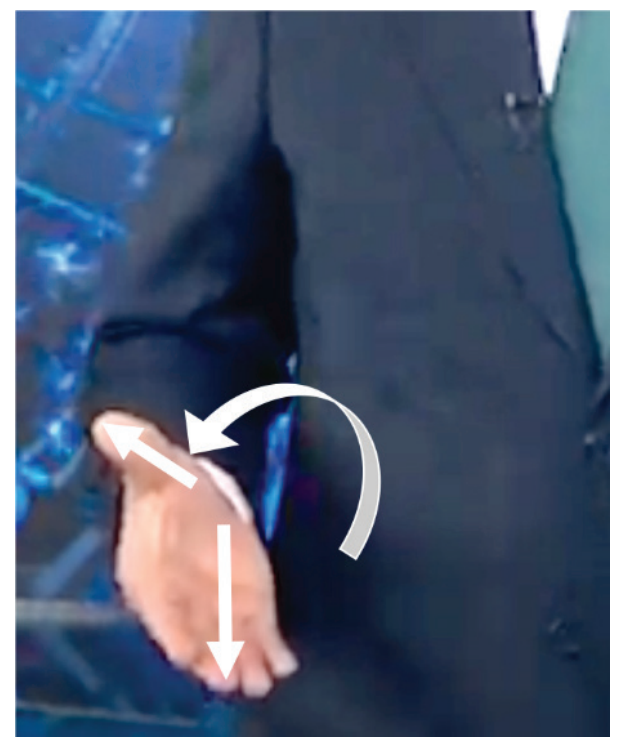

Figure 12. PUOH involving extension of the fingers, outward rotation of the forearm and wrist (abduction), leading to a nearly supine hand. 


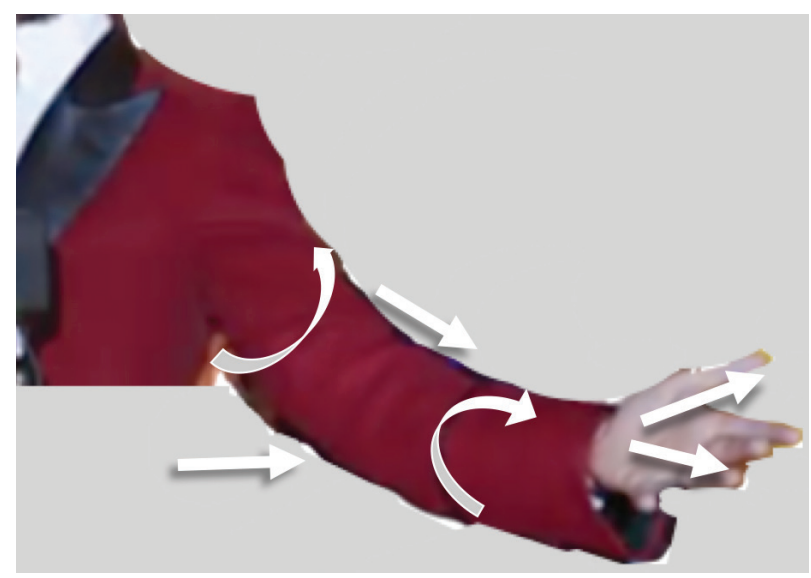

Figure 13. Exaggerated $\mathrm{PUOH}$, with extension of the fingers, rotation (abduction) of the forearm leading to supination, extension at the elbow and shoulder, and abduction of the upper arm from the shoulder.

Applying the kinesiological system here provides insights that reveal the coherence of the different gesture forms along a continuum. In the kinesiological system, the continuum constitutes a gradual adding on of bodily joints, and with them, certain degrees of freedom, and, as a consequence, the following patterns of movement:

- extension

- extension and abduction/rotation

- extension and abduction/rotation with supination

- extension and abduction/rotation with supination and extension/abduction.

However, applying the traditional four-parameter system of gesture description makes the different forms appear to be unrelated to each other, given how the parameters are realized in such different ways.

Analyzing these movements in terms of the kinesiological system provides a view of the gestural movements from the inside, rather than from the outside as the traditional four parameters do. The kinesiological interpretation shows how for the gesturer, each of these types along the continuum could be perceived as some degree of doing some version of the same thing, from a more miniature (finger lift) to a more maximal ("taa-daa" PUOH) form of expression. Using one of the smaller forms in a given moment might be seen (by the producer or by someone paying attention to them) as metonymically producing a miniature version of a fuller performance of one of the larger forms. They all form part of a continuum of degrees of exertion of effort, manifested by use of an increasing number of joints and degrees of freedom of movement.

The discussion of these various forms as different realizations of presentation gestures already indicates a common function that they serve, namely accompanying the presentation of an idea to the listening audience, and even being the observable means that indicates that the spoken utterance is an idea being presented. However, as mentioned briefly above, and as the following section shows in more detail, different scholars have not agreed in their characterization of this function. Is there a way in which their disparate analyses of function can be brought together, in line with the coherence of the analysis of forms, presented above?

\section{Analysis of the functions}

Not only do the traditional four parameters of gesture form sometimes hide commonalities between gestures that are related in their means of production, but the traditional methods of characterizing gesture functions also result in a kind of dilemma when we use them to try to capture the similarities and differences between the functions of gestures such as the four points on the continuum described above. This is mainly a consequence of the goal in much gesture research of trying to categorize gestures according to one main function for each gesture phrase (particularly the main gesture stroke plus any subsequent hold of the final position of the stroke).

The kind of presentation gesture that has been analyzed the most frequently is the $\mathrm{PUOH}$, making it the presentation gesture par excellence. However, even this type has been argued to have different functions. McNeill (1992) picked up on Reddy's (1993/1979) analysis of our language about language, and how (in English and many other languages) we frame communication as a process of putting ideas into words (as if they were containers) and sending them to recipients, e.g. phrases like getting one's idea across, or characterizing a poem as being loaded with meaning. McNeill borrowed Reddy's characterization of this as the conduit metaphor for communication and labelled gestures in which the speaker appears to hold an idea that they are talking about (through open, slightly cupped hands, either facing each other or with palms turned up) as metaphoric gestures. Müller (2004, p. 233) shows how this verbal metaphor is realized in gesture use: "The Palm Up Open Hand presents an abstract discursive object as [if it were] an inspectable one-an object which is concrete, manipulable, and visible, and it invites participants to take on a shared perspective on this object." In a similar vein, Streeck's (2009) uses the term "speech-handling" in reference to the PUOH and related gestures, further employing this metaphor in the naming of this gestural function. Bavelas et al. (1992) also acknowledged this metaphoric use of the PUOH, but claim that, in light of the range of uses of this gesture, it is better characterized as an interactive gesture. Kendon (2004, p. 158) labels it a pragmatic gesture, since it "relate[s] to features of an utterance's meaning that are not a part of its referential meaning or propositional content." Müller's (2004) paper, which is devoted exclusively to the PUOH, is one work which points out how the different variant ways in which the PUOH is produced can intensify one of these meanings or another. 
What the gestures involved in presenting a point that are discussed in this article have in common is that they function in the liminal semantic space of what Freedman (1972) calls "object-focused movements". Freedman exploits the vagueness of the word "object" in a productive way. $\mathrm{He}$ characterized the category as follows: "These movements are object-focused in two senses: they are linked to the object of one's verbally expressed experiences, the objects of representation [...]; but they are also linked to the effort to reach the listener, the object of presence" (p. 158, emphasis in original). That is: all of these gestures relate, to varying degrees, both to the idea being presented and to the audience/addressee that it is being presented to.

Some works do explicitly approach manual gestures as being inherently multifunctional (e.g. McNeill, 2005; Müller, 1998b). However, this theoretical position is rarely carried over and applied in coding schemes and analyses, with researchers tending to identify one function per gesture stroke as being primary, and therefore not discussing the secondary or tertiary functions. This is partly because of the desire to achieve greater reliability in gesture coding for quantitative analyses, which can be facilitated by having fewer categories on which coders need to reach agreement.

In Iriskhanova and Cienki (2018) a system is proposed for annotating the variety of possible functions fulfilled by any given gesture (at least as far as the researcher is able to make claims about them) and the degree to which the gesture fulfilled each function. An example of its use is shown in Fig. 14, illustrating its application to the thumbs-up emblem and a 'friendship' gesture of grasping one's own two hands together, replicating the act of shaking another's hand.

This multi-vector semiotic model involves a number of parameters of properties of gestures - functions such as semanticity (degree to which the gesture refers to a referent, property, or process), metaphoricity, and indexicality, as well as the degree of the gesture's conventionality in the given culture, degree to which the speaker

\section{Multi-vector semiotic model (emblems)}

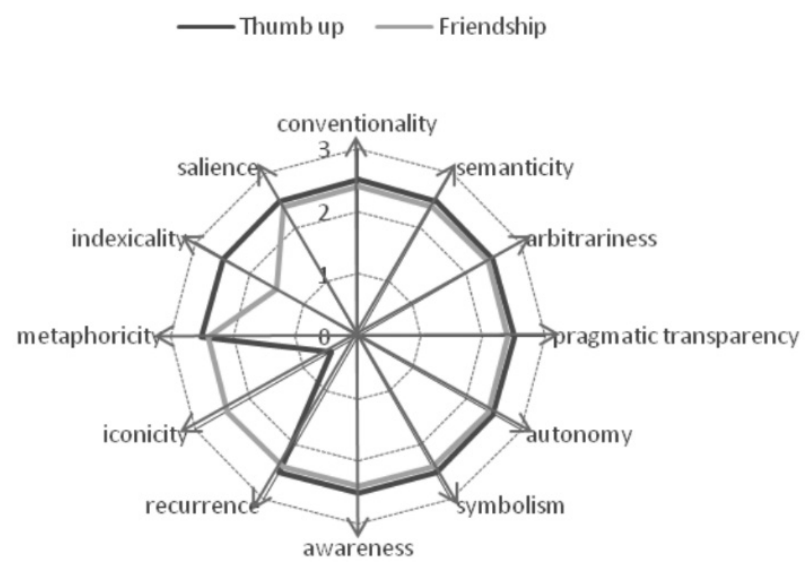

Figure 14. A multi-vector semiotic model for capturing the multifunctionality of gestures (Iriskhanova \& Cienki, 2018, p. 31). indicates awareness of having used a gesture, and the salience of the gesture in context. The particular parameters implemented in the model can be varied as appropriate for the goals of one's study (see below). Each of them is indicated along a scale which the researcher can use to evaluate the gesture phrase in context from 0 to 3 . The scales are displayed with a common starting point for 0 , radiating out from there, with the analysis of any given gesture or gestures producing a kind of spider web, as shown in Fig. 14.

Given that this system is rather complex (like gestures themselves), for the present analysis, we can limit ourselves to three of the parameters, as follows:

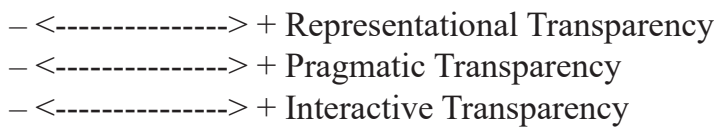

Each will be characterized in terms of how easily that function can be recognized by the researcher in the gesture in context, what will be called the transparency of the function. The method of analysis proposed here will thus not depend on claims made about what the producer of the gesture was trying to achieve (since we cannot read that person's mind to know it), but rather about what the given researcher deduces about the function, based on formal and contextual cues and their perceived salience.

The parameters to be used here are Representational Transparency (which conflates semanticity and iconicity from the multi-vector model), Pragmatic Transparency, and Interactive Transparency (a scale not taken from Iriskhanova and Cienki [2018] but one that has been introduced for this study). Each will be marked on a scale of transparency to the researcher, from less to more, indicated by the minus and plus signs.

- Representational Transparency involves the degree to which the gesture is interpreted as performing one of the Modes of Representation. Adopting the system introduced in Müller (1998a, 1998b, 2014) and adapting the terminology for the modes, we will refer to them as (a) enacting an action that would normally be done with the hands (e.g. moving one's gripped hand as if turning a handle to open a door); (b) appearing to hold or touch a surface as if it were physically present; (c) moving one or more fingertips as if drawing or tracing a form; and (d) using the hand to embody an entity, including how an entity might engage in an action (as when two extended fingers open and close, imitating the movement of the two blades of a pair of scissors). If any or several of these functions are interpreted as being involved in the gesture, this will constitute some degree of representational transparency.

- Pragmatic Transparency covers the categories noted in Kendon (2004, pp. 158-159): (a) mod- 
al functions, in which "the gesture alters in some way the frame in terms of which what is being said in the utterance is to be interpreted", which can include showing one's stance towards what is being talked about; (b) performative functions, which can indicate the speech act that the speaker is engaging in with the utterance (as in making a statement, raising a question, etc.); and (c) parsing functions, when gestures are used "as if they [were] punctuating the spoken discourse, or as if they [were] marking out its different logical components" (with rhythmic beat movements for emphasis being an example).

- Interactive Transparency concerns here the degree to which the gesture displays attention to the interlocutor. This could be through a deictic move in the direction of the listener or audience (a real or imagined addressee, and in the latter case, the direction is less relevant than the quality of being a gesture pointing outward), but this need not necessarily be a pointing gesture, per se.

What we find, ironically, is that the determination of these functions in fact takes us back to the form parameters seen from an 'external perspective', as discussed earlier. In this regard, the four form parameters prove their usefulness for this analysis, providing an entryway to move from form (as perceived from an external perspective) to function. This is actually the basis of the Linguistic System of Gesture Analysis, described in Bressem et al (2013).

The logic for choosing the three parameters described above can be traced back to Bühler's (1934) Organon model. Bühler argued that linguistic signs involve three functions at once: representation (Darstellung) of the object or states of affairs being mentioned, expression (Ausdruck) on the part of the speaker/'sender' of the message, and appeal to the listener/receiver (Appell). Müller (1998b) makes the case that each of the three functions that Bühler claimed for language could also be found in gesture. (See Müller, 2013 for a detailed explication of this claim.) In the present analysis, while the representational function carries over in a more straightforward way, Bühler's function of 'expression' is captured in the scale of Pragmatic Transparency, and that of 'appeal' plays out in the scale of Interactive Transparency.

Each parameter can be marked for the degree to which the given function is seen as being saliently transparent (to the researcher) for the given gesture phrase in its context of use. The following are suggestions of how such an analysis would look. The system can be used in at least two ways: one would be to help illustrate a given researcher's interpretive analysis of a small set of gestures as used in context (as will be done here), and the other would involve empirical testing of the system itself with multiple coders to ascertain the degree to which this system could be used for replicable analyses.
Let us consider first how each function is manifested in each of the four reference points on the scale of presentation gestures in terms of its transparency. We will identify each of them using the kinesiological characteristics, explicated above, namely:

- extension

- extension and abduction/rotation

- extension and abduction/rotation with supination

- extension and abduction/rotation with supination and extension/abduction.

\section{Representational transparency}

The function of representation in gesture is more transparent when greater effort is being applied to any given articulator(s). Greater effort in the formation of the handshape in the gesture results in a clearer manifestation of representing something. Increased tension in the bending or the straightening out of the fingers produces more clearly articulated forms. This can be opposed to when muscles in the hands are relaxed, which can result in a lack of clarity as to whether a particular form is being represented or not, given that a relaxed hand with fingers slightly bent inward is the default configuration of the hand when not gesturing.

Reference to the physiological property of tension (versus laxness) is quite informative in combination with the modes of representation for determining the degree of transparency of representation in a gesture: greater tension in the handshape can make it clearer that the form of the hand itself is being used to depict features of a referent; greater tension in the arms, resulting in elevation of the hands to a space higher up in front of the speaker, increases the salience of representational qualities that a gesture may have. (See also Cienki \& Mittelberg, 2013 and Müller, 2008 on qualities of gesture production that highlight their symbolic function in context.) In light of this, the qualification of musculature as tense versus lax is a distinction which could be useful to add to the toolkit of the kinesiological framework of analysis.

Let us consider each of the four gesture forms under study here in terms of their representational transparency. In each case, a vertical bar '|' will be used to propose the relevant point on the $-/+$ scale for that gesture form in the relevant context of use, introduced earlier.

Extension (Figs 4, 10)

-<-----|----------> + Representational Transparency

In terms of the modes of representation, the raised finger when presenting an idea can be seen as an instance of minimal embodying of the idea. For comparison, see the practice in many cultures of counting on one's fingers when naming ideas (an instantiation of the metaphor of Ideas as Objects [Sweetser, 1998]). Another interesting comparison is the handshape with the index finger ex- 
tended straight upward and the other fingers held closed in a fist, used in American Sign Language; glossed in English as UNDERSTAND, Wilcox (2000) analyzes it as reflecting the metaphor that Ideas in Existence Are Straight, with the finger embodying the idea.

Extension and abduction/rotation (Figs 3, 11) -<--|--------------> + Representational Transparency

This form constitutes a less transparent case of representation. The hand is relatively relaxed in its internal form (not tense) and therefore it is less obvious that it is engaging any one of the four modes of representation. For example, the closest mode that one might attribute to it is the holding mode, but a relaxed hand turning out and going back is not a very transparent rendition of holding something.

Extension and abduction/rotation with supination (Figs 2, 12)

$$
\text { -<-------------|--> + Representational Transparency }
$$

By contrast with simple extension and rotation, this gesture places the hand in a palm-up, fairly flat position, requiring more effort for the extension of the fingers. As the analyses of the PUOH mentioned above argue, this bears strong similarity to the hand configuration when one holds something small on one's hand. The representational transparency of this gesture is therefore fairly high, with the hand as if holding a small object.

Extension and abduction/rotation with supination and extension/abduction (Figs 1, 13)

$$
\text { -<-------------|-> + Representational Transparency }
$$

This reinforces the representation of holding something out, discussed above, by placing it in a space more external to the speaker, as one would do when physically presenting something to someone else.

\section{Pragmatic transparency}

Extension (Figs 4, 10)

$$
-<--\mid------------->+ \text { Pragmatic Transparency }
$$

Of the four gestures on this continuum, the extended finger provides the least expression of any of the pragmatic functions. However, its place on this scale, as with all of these four gestures, would move more to the positive side if produced with a conspicuous beat movement, adding emphasis to its production.

Extension and abduction/rotation (Figs 3, 11)

-<------|---------> + Pragmatic Transparency

This gesture more obviously involves adding emphasis than a smaller movement (the extension alone) just involving one finger would, especially if it is produced with prosodic stress in the speech.

Extension and abduction/rotation with supination (Figs 2, 12)

$$
\text { -<-------------|-->+ Pragmatic Transparency }
$$

The pragmatic functions of the PUOH are well known from the literature, cited previously. Kendon (2004, p. 159) actually uses the PUOH as the prime example of a gesture fulfilling a pragmatic function by performing a speech act, as it is "used as a way of indicating that what the speaker is saying is being 'offered' to the interlocutor" for consideration.

Extension and abduction/rotation with supination and extension/abduction (Figs 1, 13)

-<-------------|-> + Pragmatic Transparency

The external position of this gesture type more conspicuously adds emphasis to what is being talked about and presented, increasing its pragmatic transparency.

\section{Interactive transparency}

$$
\begin{aligned}
& \text { Extension (Figs 4, 10) } \\
& \quad-<--\mid----------->+ \text { Interactive Transparency }
\end{aligned}
$$

The extension of one finger constitutes a minimally interactive appeal; in addition, the movement may not necessarily even be visible to an interlocutor. It may seem counterintuitive to talk about a gesture's function as presenting a point and at the same time describe it as being minimally interactive, but this "points to" the bigger question of why we use gesture. Here it can be not only to present an idea to others, but also to realize for ourselves the presentation of an idea (i.e. to realize that we are presenting an idea): the imagined interactive situation can be internalized. Witness that the speaker in this example is an interpreter with no actual audience being spoken to. The gestures can be for the speaker himself, for an imagined audience, or (as discussed in Cienki \& Iriskhanova, 2020) as part of a blended viewpoint, enacting what the original speaker might have done while speaking. Note that the interpreter only heard the lecturer's voice in an audio recording and did not see him, and therefore was not reproducing any gestures he had seen.

Extension and abduction/rotation (Figs 3, 11)

- <---------|------> + Interactive Transparency

This gesture makes a small degree of appeal to the interlocutor through its movement outward, from self to other.

Extension and abduction/rotation with supination (Figs 2, 12)

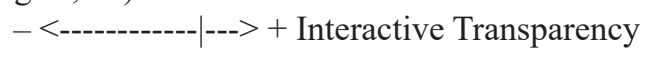


The PUOH is the prototypical example of what Bavelas (1994, p. 212) calls a gesture "marking delivery of new information for the interlocutor". In the example in Fig. 2B we see an instance where it introduces a rhetorical question, 'then what is interference?'

Extension and abduction/rotation with supination and extension/abduction (Figs 1, 13)

$$
\text { -<---------------|> + Interactive Transparency }
$$

This gesture makes a maximal appeal to the interlocutor, directed towards the audience in a peripheral area of the speaker's gesture space.

\section{Summary}

This yields the following functional profile for each gesture:

$$
\begin{aligned}
& \text { Extension (Figs 4, 10) } \\
& -<----\mid--------->+ \text { Representational Transparency } \\
& -<--\mid------------>+ \text { Pragmatic Transparency } \\
& -<--\mid------------>+ \text { Interactive Transparency }
\end{aligned}
$$

The minimal exertion with the mere extension of a finger in this gesture appears to accomplish a minimal function, expressing what Lakoff and Johnson (1980) might call an ontological metaphor-but in gesture, as the abstract became concrete, with the idea being presented verbally being realized in the minimal presentation of a finger. The small size of this action makes for minimal transparency of any pragmatic or interactive function.

Extension and abduction/rotation (Figs 3, 11)

$$
\begin{aligned}
& -<--\mid------------->+ \text { Representational Transparency } \\
& -<-----\mid--------->+ \text { Pragmatic Transparency } \\
& -<---------\mid------>+ \text { Interactive Transparency }
\end{aligned}
$$

The representational value of this hand turn-out is less clear than it is in any of the other three gestures, but the rotation outward from the self gives it a modest appearance of making an appeal via a low-effort presentation.

Extension and abduction/rotation with supination (Figs 2, 12)

$$
\begin{aligned}
& -<-------------\mid-->+ \text { Representational Transparency } \\
& -<-------------\mid-->+ \text { Pragmatic Transparency } \\
& -<-------------\mid--->+ \text { Interactive Transparency }
\end{aligned}
$$

With the rotation being sufficiently extensive so as to supinate the hand, combined with the extension of the fingers, revealing the palm, this gesture has a clearer representational function of as-if holding something on one's open hand, realizing the pragmatic (but not verbalized) function of offering, interactively appealing to the interlocutor.

Extension and abduction/rotation with supination and extension/abduction (Figs 1, 13)

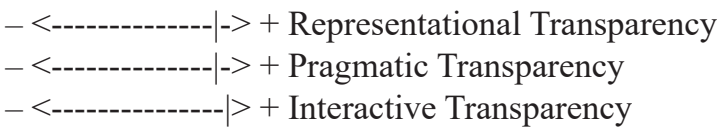

Movement of the above gesture via abduction to a more external space, oriented toward the addressee(s), adds to the transparency of all of the gesture's functions, particularly on the interactive plane.

In sum, the stepwise increase in degrees of freedom of movement that are used, as defined in the kinesiological system, makes for increasingly greater transparency of fulfilling the functions on each of the functional scales as we move along this gestural continuum.

\section{Conclusions}

Regarding the continuum of gestures presenting a point, in many respects, it is perhaps the hand turn-out that should be seen as constituting the prototype of the gestures for presenting a point, rather than the PUOH. Physiologically, witness the fundamental role of the rotation in the production of the turn-out and the $\mathrm{PUOH}$, and the fact that many instantiations of the $\mathrm{PUOH}$ do not rotate far enough to produce a truly upward facing palm; in many contexts, producing a truly supine hand would involve too much effort, would be uncomfortable (because of the degree of torque required), would not be feasible in the physical context (items worn around the wrist or objects in the environment might inhibit this degree of movement), or might be pragmatically strange (the truly supine hand might seem to be an over-exaggerated gesture). Functionally, the turn-out can often serve the same purpose as the PUOH, but with less effort. It might be seen as a metonymic enactment of a $\mathrm{PUOH}$, a part standing for the fully fledged production, and of course various gradations of the turn-out and of all the four forms presented here occur along the continuum.

We see here the value of combining both internal (kinesiological) and external (four form parameter) perspectives in gesture analysis. The internal point of view revealed how the difference between the four gestures is a matter of adding bodily joints that are moving, with their inherent possible degrees of freedom of movement. The external perspective proved most useful as a basis for grounding the interpretations, about functions in context, on observable phenomena.

The analysis proposed here can help make sense of the experiential basis of these different types of gestures as having connections to each other, as constituting different forms of presenting a point. We see this in the coherent links between the different forms and between the transparency of implementation of the different sets of functions. The kinesiological system helps reveal how the different degrees of intensity in terms of effort required to produce the forms relate to different degrees of strength in terms of the transparency of the gestures' perceived functions. In addition, the analysis of these forms 
as points on a continuum has theoretical implications. It demonstrates how what might just seem to be different gestures on the surface actually have a coherence with each other if viewed from a different perspective; that is namely the perspective of the kinesiological system of movement combined with a scalar approach to analyzing the transparency of the gestures' multiple functions. Taken together, the exemplars analyzed above constitute a category: a continuum of different ways of presenting a point, with each way entailing a different degree of prominence of doing so. This prominence is constituted for the producer in terms of the degree and kind of effort (based on the degrees of freedom) involved in the production of any given instantiation, and for the perceiver in terms of visual salience through the amount of articulators involved and the amount of gesture space used.

The form/function pairings identified can provide reference points on a continuum that can be useful for coding in other studies in the future. Coding gesture functions along continua does present a challenge in terms of assessing the reliability with which this system can be applied. This remains an issue for future research and development. However, what may help in applying the continua proposed is the framing of them in terms of transparency of the given functions to us as researchers, rather than in terms of claims about what speakers were doing with their gestures in the moment. This framing of the functions in this way, and viewing them in terms of degrees of transparency, are steps which can help further develop the Linguistic Annotation System for Gestures (Bressem et al., 2013), whose goal it is to help researchers move from the analysis of observable forms to the interpretation of gestures' functions in context.

\section{Acknowledgements}

The research for this study was supported by Russian Science Foundation grant number 19-18-00357, awarded to the PoliMod Lab of Moscow State Linguistic University. Permission to reprint Figures 6-9 (originally Figure 5.1 from the book Aspectuality across languages https:// benjamins.com/catalog/hcp.62) was kindly provided by John Benjamins Publishing Company. I wish to thank Paloma Opazo and two anonymous reviewers for their comments on the first draft. And I am (and the field of gesture studies is) greatly indebted to Dominique Boutet, who we lost to Covid-19 in May 2020. His valuable insights, his human approach to science, and his sense of humor will remain with those who had the pleasure of knowing him.

\section{References}

Bavelas, J. B. (1994). Gestures as part of speech: Methodological implications. Research on Language \& Social Interaction, 27(3), 201221. https://doi.org/10.1207/s15327973rlsi2703_3
Bavelas, J. B., Chovil, N., Lawrie, D. A., \& Wade, A. (1992). Interactive gestures. Discourse Processes, 15(4), 469-489. https://doi. org/10.1080/01638539209544823

Boutet, D. (2010). Structuration physiologique de la gestuelle: Modèle et tests. Lidil, 42, 77-96. https://doi.org/10.4000/lidil.3070

Boutet, D. (2018). Pour une approche kinésiologique de la gestualité: Synthèse. Unpublished habilitation, Université de Rouen-Normandie.

Boutet, D. (to appear). A kinesiological approach to gesture analysis. In A. Cienki (Ed.), The Cambridge handbook of gesture studies. Cambridge: Cambridge University Press.

Boutet, D., Morgenstern, A., \& Cienki, A. (2016). Grammatical aspect and gesture in French: A kinsiological approach. Russian Journal of Linguistics, 20(3), 132-151.

Boutet, D., Morgenstern, A., \& Cienki, A. (2018). Looking ahead: Kinesiological analysis. In A. Cienki \& O. K. Iriskhanova (Eds.), Aspectuality across languages: Event construal in speech and gesture (pp. 143-159). Amsterdam: John Benjamins.

Bressem, J. (2013). A linguistic perspective on the notation of form features in gestures. In C. Müller, A. Cienki, E. Fricke, S. Ladewig, D. McNeill, \& S. Teßendorf (Eds.), Body - Language - Communication. Volume 1 (pp. 1079-1098). Berlin: De Gruyter Mouton.

Bressem, J., Ladewig, S. H., \& Müller, C. (2013). Linguistic annotation system for gestures (LASG). In C. Müller, A. Cienki, E. Fricke, S. Ladewig, D. McNeill, \& S. Teßendorf(Eds.), Body-language-communication. Volume 1 (pp. 1098-1124). Berlin: De Gruyter Mouton.

Bressem, J., \& Müller, C. (2014). A repertoire of German recurrent gestures with pragmatic functions. In C. Müller, A. Cienki, E. Fricke, S. Ladewig, D. McNeill, \& J. Bressem (Eds.), Body-language-communication. Volume 2 (pp. 1575-1591). Berlin: De Gruyter Mouton.

Bühler, K. (1934). Sprachtheorie: Die Darstellungsfunktion der Sprache. Jena: Gustav Fischer.

Calbris, G. (2011). Elements of meaning in gesture. (Translated by M. M. Copple.) Amsterdam: John Benjamins. https://doi.org/10.1075/gs.5

Cienki, A. (2013). Gesture, space, grammar, and cognition. In P. Auer, M. Hilpert, A. Stukenbrock, \& B. Szmrecsanyi (Eds.), Space in language and linguistics: Geographical, interactional, and cognitive perspectives (pp. 667-686). Berlin: Walter de Gruyter.

Cienki, A., \& Iriskhanova, O. K. (2020). Patterns of multimodal behavior under cognitive load: An analysis of simultaneous interpretation from L2 to L1. Voprosy Kognitivnoy Lingvistiki, 1, 5-11. https://doi. org/10.20916/1812-3228-2020-1-5-11

Cienki, A., \& Mittelberg, I. (2013). Creativity in the forms and functions of spontaneous gesture with speech. In T. Veale, K. Feyaerts, \& C. Forceville (Eds.), The agile mind: A multi-disciplinary study of a multi-faceted phenomenon (pp. 231-252). Berlin: De Gruyter Mouton.

Cooperrider, K., Abner, N., \& Goldin-Meadow, S. (2018). The palmup puzzle: Meanings and origins of a widespread form in gesture and sign. Frontiers in Communication, 3, Article 23. https://doi. org/10.3389/fcomm.2018.00023

Efron, D. (1972). Gesture, race, and culture. The Hague: Mouton. (First published 1941)

Ferré, G. (2011). Functions of three open-palm hand gestures. Multimodal Communication, 1(1), 5-20. https://doi.org/10.1515/mc-2012-0002

Freedman, N. (1972). The analysis of movement behavior during the clinical interview. In A. W. Siegman \& B. Pope (Eds.), Studies in dyadic communication (pp. 153-175). New York, NY: Pergamon Press. https://doi.org/10.1016/B978-0-08-015867-9.50012-5 
Grishina, E. A. (2017). Russkaja zhestikuljacija s lingvisticheskoj tochki zrenija: Korpusnye issledovanija [Russian gesticulation from a linguistic perspective: A collection of corpus studies]. Moscow: Languages of Slavic Culture.

Iriskhanova, O. K., \& Cienki, A. (2018). The semiotics of gestures in cognitive linguistics: Contributions and challenges. Voprosy Kognitivnoy Lingvistiki, 4, 25-36. https://doi.org/10.20916/1812-32282018-4-25-36

Kapandji, I. A. (1997). Physiologie articulaire. 1, Membre supérieur. Paris: Maloine.

Kendon, A. (1980). Gesticulation and speech: Two aspects of the process of utterance. In M. R. Key (Ed.), The relation between verbal and nonverbal communication (pp. 207-227). The Hague: Mouton. https://doi.org/10.1515/9783110813098.207

Kendon, A. (2004). Gesture: Visible action as utterance. Cambridge: Cambridge University Press. https://doi.org/10.1017/CBO9780511807572

Kohler, K. J. (2018). Communicative functions and linguistic forms in speech interaction. Cambridge: Cambridge University Press. https:// doi.org/10.1017/9781316756782

Laban, R., \& Lawrence, F. C. (1974). Effort: Economy of human movement. London: Macdonald \& Evans. (First published 1947)

Lakoff, G., \& Johnson, M. (1980). Metaphors we live by. Chicago: University of Chicago Press.

McNeill, D. (1992). Hand and mind: What gestures reveal about thought. Chicago: University of Chicago Press.

McNeill, D. (2005). Gesture and thought. Chicago: University of Chicago Press. https://doi.org/10.7208/chicago/9780226514642.001.0001

Müller, C. (1998a). Iconicity and gesture. In S. Santi, I. Guaïtella, C. Cavé, \& G. Konopczynski (Eds), Oralité et gestualité: Communication multimodale, interaction (pp. 321-328). Paris: L'Harmattan.

Müller, C. (1998b). Redebegleitende Gesten. Kulturgeschichte-Theorie - Sprachvergleich. Berlin: Berlin Verlag A. Spitz.

Müller, C. (2004). Forms and uses of the Palm Up Open Hand: A case of a gesture family? In C. Müller \& R. Posner (Eds), The semantics and pragmatics of everyday gestures: The Berlin conference (pp. 233-256). Berlin: Weidler Buchverlag.

Müller, C. (2008). What gestures reveal about the nature of metaphor. In A. Cienki \& C. Müller (Eds.), Metaphor and gesture (pp. 219-245). Amsterdam: John Benjamins. https://doi.org/10.1075/ gs.3.12mul

Müller, C. (2010). Wie Gesten bedeuten. Sprache und Literatur, 41(1), 37-68. https://doi.org/10.1163/25890859-041-01-90000004

Müller, C. (2013). Gestures as a medium of expression: The linguistic potential of gestures. In C. Müller, A. Cienki, E. Fricke, S. Ladewig, D. McNeill, \& S. Teßendorf (Eds.), Body-language-communication. Volume 1, (pp. 202-217). Berlin: De Gruyter Mouton.

Müller, C. (2014). Gestural modes of representation as techniques of depiction. In C. Müller, A. Cienki, E. Fricke, S. Ladewig, D. McNeill, \& J. Bressem (Eds.), Body-language - communication. Volume 2 (pp. 1687-1701). Berlin: De Gruyter Mouton.

Quintillian, M. F. (1922). The Institutio Oratoria of Quintilian with an English translation by H. E. Butler. New York, NY: G. P. Putnam and Sons.

Reddy, M. (1993). The conduit metaphor: A case of frame conflict in our language about language. In A. Ortony (Ed.), Metaphor and thought (pp. 164-201) (second edition). Cambridge: Cambridge University Press. (First published 1979) https://doi.org/10.1017/ CBO9781139173865.012

Stokoe, W. C. (1960). Sign language structure: An outline of the visual communication systems of the American deaf. Buffalo, NY: Dept. of Anthropology and Linguistics, University of Buffalo.

Streeck, J. (2009). Gesturecraft: The manu-facture of meaning. Amsterdam: John Benjamins. https://doi.org/10.1075/gs.2

Sweetser, E. (1998). Regular metaphoricity in gesture: Bodily-based models of speech interaction. Actes du 16 e Congrès International des Linguistes, (CD-ROM). Elsevier.

Wilcox, P. P. (2000). Metaphor in American Sign Language. Washington, DC: Gallaudet University Press. 